\title{
Themakarten - Möglichkeiten im Unterricht
}

\author{
Es gibt viele Anleitungen zum Kartenlesen. Sie auch, dass Themakarten ebenso Arbeitsinstru- \\ befassen sich ebenso wie der Unterricht im ment wie Anschauungsmaterial sind. Gleich- \\ Kartenlesen ausschliesslich mit topographischen zeitig erzieht er den Schüler zur Genauigkeit \\ Karten. Das Lesen von Themakarten wird kaum im Ausdruck.
} je systematisch beschrieben und geübt. Darum haben manche Lehrer Mühe, durch gezielte Fragestellungen Themakarten als Grundlage für weitere Ueberlegungen zu verwenden. In zwei Beiträgen sollen dem Lehrer - es ist dabei in erster Linie an Mittelschullehrer gedacht die mittlere und obere Klassen unterrichten - Anregungen in dieser Richtung gegeben werden. Der Verlag K\&F stellte der Geographi- L ca Helvetica zwei Kartenblätter aus "Wirtschaftsgeographischer Weltatlas" zur Verfügung(1). Die Fragen wurden im Kreise der Assistenten am Geographischen Institut der Universität Zürich besprochen. Der Text zu Karte (I) wurde von H. Boesch, jener zu Karte (II) von $M$. Tschanz verfasst. Unterschiede im Stil und in der Fragestellung wurden mit voller Absicht nicht ausgeglichen.

Beide Kartenbeispiele gehören jener Kategorie von Themakarten an, welche wir quantitative. nennen könnten. Das Dargestellte wird in einem bestimmten Mass gemessen und jeder Signatur eine bestimmte Menge zugeschrieben. Der Schüler sollte, wenn er eine vom Lehrer entsprechend formulierte Frage an die Karte weitergibt, dort eine eindeutige Antwort finden. Der Schlüssel dazu ist die Legende, die als erstes sorgfältig studiert sein will.

Die folgenden Beispiele beginnen grundsätzlich mit einer Frage (Aufgabe). Oft schliesst sich

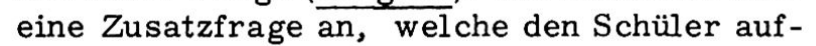
fordert, weitere Informationen im Schweizerischen Mittelschulatlas (MA) zu suchen. Mit den auf diese Weise erarbeiteten Tatsachen können anschliessend die als Beispiele aufgeführten Themen a, busw. besprochen werden Eine sinnvolle Besprechung der Themen stellt oft beträchtliche Anforderungen an den Lehrer, weil er aus Literatur, Zeitungen usw. umfangreiches Tatsachenmaterial zusammenstellen muss. Mit solchen Aufgaben erwirbt der Schüler auf zwanglose Weise auch eine solide Kenntnis der sogenannten "Briefträgergeographie". Im uebrigen wird jeder Lehrer in der Lage sein, weitere Fragen und Themen zu formulieren. Indem er sie in der angegebenen Weise im Unterricht verwendet, zeigt er dem Schüler

1. Tee, Kaffee, Kakao, Zucker

Aufgabe: Für Indien und Kuba sind tabellarisch die Gewichtsmengen Zuckerrohr und Rohrzucker und der errechnete Prozentanteil des Rohrzuckers am Zuckerrohr anzugeben. Den Unterschied von zentrifugiertem und nicht-zentrifugiertem Zucker (siehe Legende) erklärt der Lehrer.

a. Unter Beachtung der Regeln über Auf - und Abrunden ist die Genauigkeit des errechneten Prozentanteiles zu untersuchen.

b. Mögliche Gründe für den Unterschied beim Prozentanteil sind zusammenzustellen. Prüfe anschliessend, welche auszuscheiden und welche als möglich anzunehmen sind. ( $\mathrm{Na}$ türliche Anbaubedingungen z. T. nach MA, Zuckerrohrsorten, Technik der Zuckergewinnung).

c. Nach den Statistiken der FAO ergibt sich für Indien ein Anteil von nicht-zentrifugiertem Zucker (in \% der totalen Zuckerproduktion von $1961 / 65=68,1970=60,1971=64$ und $1972=77$. Für Kuba wird der Wert 0 angegeben. Das Verhältnis Zuckerrohr : Zucker betrug in den genannten Jahren in Indien: 8. 8, 8. 7, 9.2 und 9.1, in Kuba: 12.4, 10. 5, 11. 0 und 9.8. Wie können diese Angaben interpretiert werden?

Bemerkung: Der Lehrer sollte an irgend einem Beispiel illustrieren, dass bei der Aufnahme von statistischen Daten oft grobe Ungenauigkeiten resultieren können.

Aufgabe: Die Antworten verlangen einen Vergleich der vorliegenden Karte mit zahlreichen Karten des MA. (a) Wo wird Zuckerrohr ausserhalb der Wendekreise angebaut ? Zusammenstellen von Klimadaten für diese Gebiete. Welcher Klimawert korreliert besonders hoch mit der äussersten Grenze des Anbaues von Zuckerrohr? (b) In gleicher Weise soll versucht werden, mögliche Gründe für die Begrenzung des

Prof. Dr. H. Boesch und M. Tschanz, dipl. nat., Assistent, Geographisches Institut der Universität Zürich, Blümlisalpstr. 10, 8006 Zürich. 
europäischen Zuckerrübenanbaues zu finden.

a. Mit Hilfe der Literatur ist zu verifizieren, ob die gezogenen Schlüsse mit Bezug auf die Begrenzung des Zuckerrohranbaues richtig waren:

b. Anschliessend ist zu prüfen, ob sich nicht auch anderswo klimatische Bedingungen finden, wie sie für die Rübenzuckergebiete in Europa festgestellt wurden.

c. Besprechen von weiteren Gründen für die Konzentration des Anbaues von Zuckerrüben in Europa; Illustrierung mit Beispielen aus der Schweiz. (Historische, wirtschaftspolitische, Fruchtwechselwirtschaft, Silage und Rübenschnitzel als Futtermittel usw.)

Aufgabe: Vorerst erklärt der Lehrer den Begriff Nettoimport (Legende). Aufgrund der Karte wird vorerst eine Liste aller Länder mit Nettoimporten aufgestellt und anschliessend nach den Grundsätzen der Klassenintervallbildung eine Gruppenbildung vorgenommen.

a. Zuckerbilanz eines Landes: Genügen Angaben über Produktion, Einfuhr und Ausfuhr zur Berechnung? Wo findet man Unterlagen, welche darüber sowie über Veränderung der Lagerhaltung, Verluste usw. Auskunft geben?

b. Welche Bedeutung kommt dem Wert "Zukkerverbrauch je Einwohner" zu? Vergleiche im Stat. Jahrb. d. Schweiz den Zuckerverbrauch für Arbeiter und Angestellte.

c. Welche Variabeln könnten das Kartenbild ändern, ohne dass sich die importierten Zuckermengen ändern (Zuckerpreis, Wert des US Dollars)?

Aufgabe: Welche Grundzüge der räumlichen Verteilung lässt der erste visuelle Eindruck der Karte für Tee, Kaffee und Kakao erkennen (Tropen, Konzentration auf einzelne Kontinente)? Diese Feststellungen sind sprachlich korrekt zu formulieren.

a. Aufstellen von Hypothesen zur Erklärung der festgestellten räumlichen Verteilung (natürliche Bedingungen, Ursprung und Verbreitung der Kulturpflanzen, koloniale Wirtschaftspolitik usw. )

b. Verifizierung diaser Hypothesen, was vom Lehrer eine beträchtliche Vorarbeit und die Beschaffung von Dokumentation verlangt.
Aufgabe: Tee, Kaffee und Kakao sind nach ihren Wärmeansprüchen zu ordnen. Es ist festzustellen, wo die Karte auf diese Frage eindeutig Antwort geben kann, und wo dies wegen ihrer starken Generalisierung nicht mehr der Fall ist.

a. Besprechung der Stockwerke der tropischen Landwirtschaft.

b. Zuordnung von Tee, Kaffee und Kakao sowie anderen Kulturpflanzen zu den entsprechenden Stockwerken.

c. Weit vom Aequator entfernte Teeanbaugebiete nach MA benennen, die klimatischen Verhältnisse feststellen und die Zusammenhänge zwischen Klimakurve und Vegetationsrhytmus für alle drei Kulturpflanzen besprechen.

Aufgabe: Beurteile vorerst rein qualitativ-visuell die Frage, welches der drei Produkte Tee, Kaffee und Kakao die stärkste Konzentration der Produktions gebiete zeigt.

a. Der eben gebrauchte Begriff "Konzentration" ist genauer zu untersuchen: Was versteht man unter Konzentration? Welche Möglichkeiten bestehen, Konzentration zu messen, um zu einer numerischen Aussage zu kommen?

b. Diskussion über qualitative und quantitative Aussagen, Vor - und Nachteile.

Aufgabe: Von den Gesamtausfuhren entfallen auf Zucker in Kuba 80-85\%, auf Kaffee in Kolumbien bis zu $60 \%$, auf Tee in Sri Lanka 50-60\% und auf Kakao in Ghana 60-70\%.

a. Wie könnte auf der vorliegenden Karte auch der Export (Nettoexport) dargestellt werden?

b. Interpretiere die erwähnten Angaben im Hinblick auf die Volkswirtschaft dieser Länder und ziehe bei der Beurteilung auch die Preisentwicklung (Handelsteil einer Zeitung, Spezialliteratur) über mehrere Jahre bei.

c. Welche Länder dürften sich nach der Karte in einer ähnlichen Situation befinden? Ueberprüfe die gezogenen Schlüsse.

(1) Boesch H. : Wirtschaftsgeographischer Weltatlas, K\&F, Bern, 1968, 1969 und 1975 letzte Auflage mit neuesten Statistiken). 
Kartenbeilage

zum Artikel H. Boesch und M. Tschanz

Themakarten - Möglichkeiten im Unterricht

Geographica Helvetica 1975, Nr.1, S. 29-30 


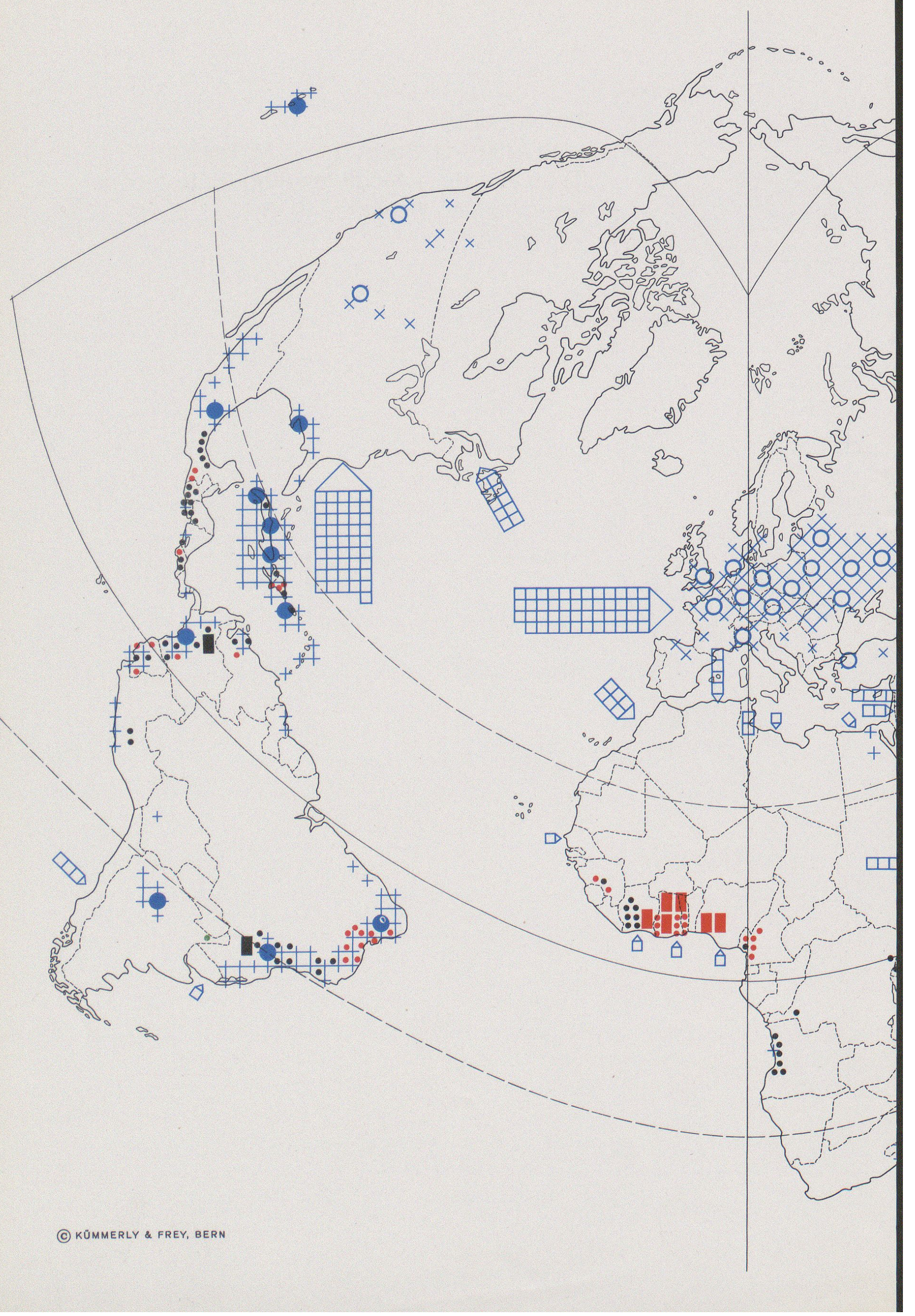


